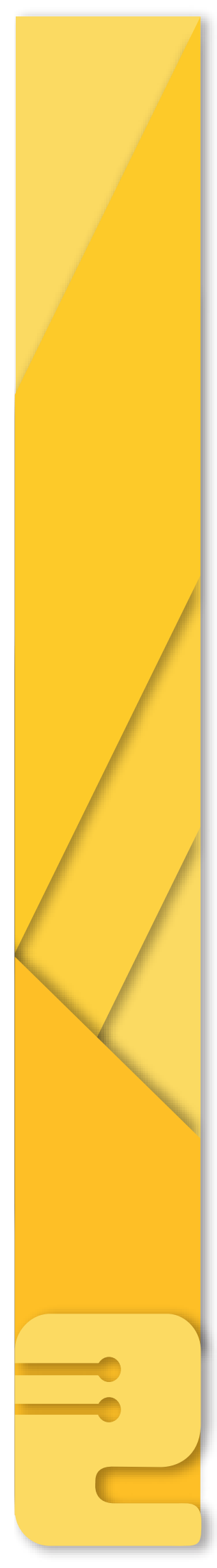

\title{
PIGMENTOS VEGETALES Y COMPUESTOS NATURALES APLICADOS EN PRODUCTOS CÁRNICOS COMO COLORANTES Y/O ANTIOXIDANTES: REVISIÓN
}

\author{
VEGETABLE PIGMENTS AND NATURAL COMPOUNDS APPLIED AS COLORANT AND \\ ANTIOXIDANTS IN MEAT PRODUCTS: REVIEW. \\ PIGMENTOS VEGETAIS E COMPOSTOS NATURAIS APLICADOS EM PRODUTOS CÁRNICOS \\ COMO COLORANTES E/OU ANTIOXIDANTES: REVISÃO \\ Jader Martínez Girón', Jorge Armando Martínez², Laura García Hurtado², Jhoan David Cuaran², \\ Yesica Alexandra Ocampo ${ }^{2}$
}

Fecha de recibido: Agosto 05 de 2016 | Fecha de Aprobado: Septiembre 24 de 2016

\section{Resumen}

La necesidad de encontrar productos naturales que tengan aplicación funcional como colorantes y/o antioxidantes en los productos cárnicos es una temática que hoy por hoy tiene una gran acogida, debido al interés social de consumir productos saludables y nutritivos. Es por esto que en este artículo de revisión se presentan algunas de las principales investigaciones que se han adelantado a nivel nacional e internacional sobre esta temática de estudio. Es de resaltar que a la fecha existen un gran número de productos y subproductos vegetales que son tema de investigación como fuente de colorantes y antioxidantes naturales en productos cárnicos. En dichos productos hay presencia de compuestos bioactivos de interés como lo son el licopeno, la capsantina, antocianinas, luteína, betalaínas, curcumina y entre otros como flores de litchi, hojas de M.Oleifera, extracto de zanahoria, etc.

Palabras clave: Antioxidante natural, colorante natural, extracto vegetal, embutido cárnico.

1 Docente e investigador de la Universidad del Valle, Facultad de Ingeniería, Sede Palmira; químico y tecnólogo en alimentos; magíster en educación de la Universidad Católica de Oriente; magíster en ingeniería agroindustrial de la Universidad Nacional de Colombia, Sede Palmira. Correo electrónico: jader.martinez@ correounivalle.edu.co Universidad del Valle, Sede Palmira,

2 Joven-Investigador(a) de la Universidad del Valle, Sede Palmira, Facultad de Ingeniería; estudiante del Programa Académico de Tecnología en Alimentos. 


\section{Abstract}

The necessity to find natural products to be applied as colorants and and/or antioxidants in meat products is a topic with a good reception today, due to the social interest for consuming nutritious and healthy products. For that reason, this article presents some of the main researches conducted national and internationally about this study field. It is important to emphasize that by this time, there are a large amount of vegetable products and sub products used as natural colorants and antioxidants in meat products, which are matter of research. In these products, there is presence of bioactive compounds of interest such as lycopene, capsanthin, anthocyanins, lutein, betalain, curcumin, among others as litchi flowers, leaves of M.Oleifera, carrot extract, etc.

Keywords: Natural antioxidant, natural colorant, vegetable extract, lunchmeat.

\section{Resumo}

A necessidade de encontrar produtos naturais que tenham aplicativo funcional como colorantes e/ou antioxidantes nos produtos cárneos é uma temática que hoje por hoje tem uma grande acolhida, devido ao interesse social de consumir produtos saudáveis e nutritivos. É por isto que neste artigo de revisão se apresentam algumas das principais investigações que se adiantaram a nível nacional e internacional sobre esta temática de estudo. É de realçar que à data existem um grande número de produtos e subprodutos vegetais que são tema de investigação como fonte de colorantes e antioxidantes naturais em produtos cárneos. Em ditos produtos há presença de compostos bioactivos de interesse como o são o licopeno, a capsantina, antocianinas, luteína, betalainas, curcumina e entre outros como flores de litchi, folhas de M.Oleifera, extrato de cenoura, etc.

Palavras chave: Antioxidante natural, colorante natural, extrato vegetal, embutido cárneos. 


\section{INTRODUCCIÓN}

Existe una diversa cantidad de compuestos tanto naturales como sintéticos de numerosas características que a la fecha han sido utilizados como aditivos en diferentes formulaciones en la industria alimentaria, cosmética, farmacéutica, etc. (Lazze et al., 2004). A nivel mundial, el mayor uso de colorantes en casi todos los productos fabricados que consumimos en la actualidad están asociados a pigmentos sintéticos dentro de los que se encuentran los colorantes del grupo azo (como la tartrazina) y otros grupos de compuestos heterocíclicos (Bener et al., 2010).

Sin embargo, debido a investigaciones que se han adelantado acerca de la toxicidad de pigmentos sintéticos, el uso de ellos se ha reducido en la gran mayoría de países del mundo (Jiang \& Xiong, 2016). Por lo tanto, existe un valioso interés en lograr encontrar la forma de utilizar pigmentos naturales que puedan sustituir a los sintéticos con su mismo poder funcional y con bajos costos de obtención (Jensen et al., 2011). En este sentido, algunos autores como Boo et al. (2012) manifiestan que el color es un parámetro decisivo para el consumidor, ya que es un factor que influye directamente en la calidad final del producto.

En este orden de ideas, los pigmentos vegetales incluyen una variedad de diferentes gamas de componentes y colores, donde se pueden mencionar principalmente los flavonoides, antocianinas, carotenoides, betalaínas, clorofilas, etc. (Valenzuela \& Pérez, 2016).

A nivel experimental, estos pigmentos pueden ser cuantificados al emplear diferentes técnicas analíticas e instrumentales como la espectroscopía, debido a que el grupo cromóforo de cada uno de los pigmentos vegetales puede absorber longitudes de onda que pueden variar entre $350 \mathrm{~nm}$ y $750 \mathrm{~nm}$ de la luz visible dependiendo de la naturaleza química del analito (Singh et al., 2005).

\section{PIGMENTOS VEGETALES}

Los pigmentos vegetales se pueden clasificar generalmente en pigmentos liposolubles y pigmentos hidrosolubles (Zhoh et al., 2010). Dentro del grupo de los pigmentos liposolubles se encuentran principalmente las clorofilas y los carotenoides, y dentro del grupo de los pigmentos hidrosolubles se encuentran las antocianinas, las betalaínas y los flavonoides (Valenzuela \& Pérez, 2016).
En este sentido, la clorofila es el pigmento responsable del color verde de las plantas. Se puede clasificar en clorofila a y b. Los carotenoides son tetraterpenoides responsables del color rojo, naranja o amarillo de la mayoría de plantas y frutos. Los carotenoides más conocidos son el a y B-caroteno (pigmento naranja), luteína (pigmento amarillo), y el licopeno (pigmento rojo). Los carotenoides actúan como antioxidantes y promueven la salud visual en los seres humanos al ser algunos fuentes de provitamina A (Burdulis et al., 2009; Rodríguez-Amaya, 2015).

Las antocianinas son glucósidos de antocianidinas, pertenecientes a la familia de los flavonoides que pueden presentar tonalidades rojas y/o azules. De acuerdo con el pH (Garzón, 2008), estas tonalidades se producen en algunos de los tejidos de las plantas superiores que proporcionan estos colores en las hojas, tallos de plantas, raíces, flores y frutos (Jensen et al., 2011). A su vez las antocianinas están involucradas en una amplia relación metabólica de actividades biológicas contra la enfermedad coronaria, el riesgo de cáncer y el sistema inmune (Kong et al., 2003), debido a que los pigmentos antociánicos permanecen intactos durante el paso del tracto digestivo al sistema sanguíneo aportando un efecto antioxidante contra las diferentes especies de oxígeno reactivo que inciden en las etapas de iniciación, promoción y progresión de la carcinogénesis (Garzón, 2008).

Por otra parte, las betalaínas presentan tonalidades rojas y/o amarillas, pero, a diferencia de las antocianinas, son compuestos alcaloides derivados del indol y sintetizados de la tirosina. Éstos son responsables del color rojo oscuro de la remolacha, la tuna roja y otras especies comestibles y no comestibles (Jiménez et al., 2014).

\section{PIGMENTOS USADOS COMO COLORANTES NATURALES EN PRODUCTOS CÁRNICOS}

En los últimos años se ha incrementado el consumo de productos cárnicos, los cuales llevan dentro de su formulación diferentes compuestos sintéticos como los nitratos y nitritos utilizados como agentes conservantes (Honikel, 2008). A su vez, se utiliza el aditivo artificial eritorbato de sodio con niveles de concentración de 0.02 a $5.00 \%$ p/p para darle a los productos un mejor color, evitar la oxidación e inhibir el crecimiento de los microorganismos (Barringer et al., 2005; Mercadante et al., 2010); sin 
embargo, con el paso de los años, las investigaciones en toxicología han demostrado que estos aditivos sintéticos son dañinos para la salud del consumidor por la formación de N-nitrosaminas. Dado lo anterior, existe la tendencia actual de cambiar y/o renovar la composición de estos productos mediante el uso de nuevos métodos y/o alternativas como lo son los pigmentos de origen vegetal, dado que poseen capacidad colorante y/o antioxidante, pero con la gran diferencia que no son dañinos para la salud destacándose en aplicación de productos cárnicos el licopeno, capsantina, antocianinas, luteína, betalaínas, curcumina, entre otros (Sánchez \& Rocío, 2013; Jiang \& Xiong, 2016).

\section{Licopeno :}

El licopeno (figura 1) es un componente funcional de importancia en la alimentación del ser humano (Choski \& Joshi, 2007). Este pigmento se extrae principalmente del tomate y es de la familia de los carotenoides, posee efectos antioxidantes, antiinflamatorios y quimioterapéuticos y aporta un color rojo como pigmento (Bojórquez et al., 2013). Debido a su actividad colorante, autores como Osterlie y Lerfall (2005) emplearon licopeno en la elaboración de carne procesada y obtuvieron una coloración roja en el producto final con un valor de acidez bajo. Adicionalmente dada la acidez natural del tomate, el pH en la carne fue bajo (5.1); por lo tanto, el crecimiento de microrganismos no fue significativo. Por otra parte, Deda et al. (2007) reportaron que la adición del 12\% $\mathrm{p} / \mathrm{p}$ de pasta de tomate en la elaboración de salchichas permitió la reducción de los nitritos de un nivel de concentración de 150 ppm a 100 ppm sin dar ningún efecto negativo en la calidad del producto final. Adicionalmente Calvo et al. (2008) evaluaron la adición de piel de tomate en salchichas en concentraciones de $0.6,0.9$ y $1,2 \% \mathrm{p} / \mathrm{p}$, encontrado que estos porcentajes permitieron mejorar las características sensoriales, texturales y la aceptabilidad general del producto que informó concentraciones de 0.26 y $0.58 \mathrm{mg}$ licopeno $/ 100 \mathrm{~g}$ salchicha. A su vez, García et al (2009) encontraron que la adición de piel de tomate seco para hamburguesas crudas y cocidas en cantidades de 0.6 a 10\% p/p mejoró los parámetros de color $a^{*}$ y $b^{*}$ y las propiedades de texturas, con valores de dureza de (67.6 N). Doménech-Asensi et al. (2013) evaluaron la adición de licopeno presente en pasta de tomate en la formulación de mortadela con niveles de adición de 2.6 y $10 \%$ p/p y encontraron que la adición del pigmento mejoró el color del producto con una estabilidad deseable durante la cocción y el almacenamiento.
Figura 1. Estructura del licopeno

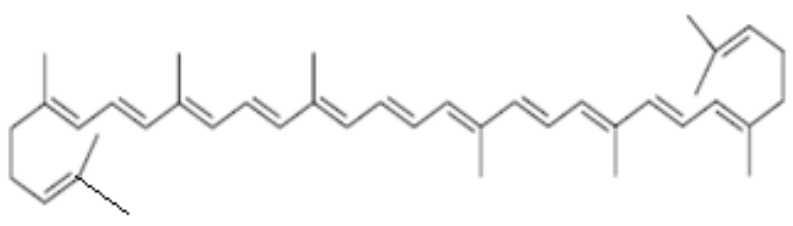

Fuente: elaboración propia.

\section{Capsantinina:}

La capsantina (figura 2) es un pigmento de color rojizo, principalmente extraído de la oleorresina del pimentón. Posee una propiedad funcional con alta actividad de color ASTA (American Spice Trade Association) en la adición de pastas, condimentos en polvo y embutidos cárnicos. Dado lo anterior, ha sido un pigmento de interés como sustituto de los colorantes artificiales. En este sentido, Fernández et al. (2002) reportaron que la adición de $30 \mathrm{~g} / \mathrm{Kg}$ de pimentón en polvo (paprika) en salchichas permitió mejorar el color rojo del producto final empleando espectrofotometría de reflectancia y coordenadas CIEL*a*b*. (Revilla \& Quintana, 2005). De la misma manera, reportaron la adición de paprika en embutidos tipo salchicha y chorizo que generó una aceleración en la maduración de la carne, además obtuvieron como resultado un crecimiento de bacterias ácido lácticas que originó un descenso rápido del $\mathrm{pH}$. Por otra parte, hubo enrojecimiento en salchichas y chorizo fresco (Gómez et al., 2008), pues evaluaron la adición de pimentón pasteurizado en cantidades de $0.3 \% \mathrm{p} / \mathrm{p}$ en salchicha roja fresca y $2 \% \mathrm{p} / \mathrm{p}$ en chorizo fresco, junto con la adición de $1 \% \mathrm{p} / \mathrm{p}$ de extracto de romero, dando resultados positivos con mayor enrojecimiento en los productos cárnicos y un aumento en la vida útil de estos productos, debido al aumento de la preservación del color. (Garzón et al., 2012).

Finalmente, elaboraron un colorante natural a partir de la oleorresina de pimentón (oLP) extraída a escala semi-industrial por fluidos supercríticos (FSC) y micro encapsulamiento por spray-drying (ORP). El colorante obtenido en polvo fue aplicado en un embutido cárnico (chorizo) que permitió la obtención de un producto con mayor tiempo de vida útil, se observó también un cambio de color menor en la muestra tratada con oleorresina extraída por FSC que con la ORP micro encapsulada. Como conclusión, los productos elaborados a partir del pimentón son una alternativa de colorante natural en productos cárnicos dependiendo del porcentaje 
de adición (\%p/p) y la tecnología de elaboración y/o procesamiento.

Figura 2. Estructura de la capsantina<smiles>CC1=C(/C=C/C(C)=C/C=C/C(C)=C/C=C/C=C(C)/C=C/C=C(C)/C=C/C=C2OC3(C)C[C@@H](O)C[C@@]2(C)C3(C)C)C(C)(C)CC(O)C1</smiles>

Fuente: elaboración propia.

\section{Antocianinas}

Las antocianinas (figura 3 ) son pigmentos que se encuentran ampliamente distribuidos en la naturaleza, son responsables del color rojo, violeta y azul característico de diferentes frutas. Éstas, a su vez, se clasifican dentro del grupo de los flavonoides (Ortíz et al., 2011; Hurtado \& Pérez, 2014). Adicionalmente, poseen propiedades antioxidantes que depende de la estructura y el pH (Cabrita et al., 2000). Dado lo anterior, estos pigmentos han sido de interés como colorantes naturales en productos cárnicos. En este sentido, Keeton et al. (2002) emplearon polvo de ciruela seca en concentraciones de 2.5 y $5 \%$ p/p en la elaboración de productos cárnicos y lograron reducir la oxidación de lípidos, prevenir el sobrecalentamiento, mejorar el color del producto final y aumentar la dulzura de la carne. Por otra parte, Selani et al. (2011) evaluaron los efectos de la adición de residuos de uva de árbol (variedad Isabel) y (variedad Niagara) en muestras de carne de pollo cruda y cocida, sobre los parámetros de color, $\mathrm{pH}$ y características sensoriales, expuestos a una temperatura de $-18{ }^{\circ} \mathrm{C}$ durante nueve meses. Estos autores informaron que los resultados sensoriales de olor y sabor fueron satisfactorios durante el almacenamiento, sin afectarse, la estabilidad del color. Adicionalmente, se disminuyó la oxidación lipídica durante la congelación.

Figura 3. Estructura de antocianinas<smiles></smiles>

Fuente: elaboración propia.

\section{Luteina}

La luteína (Figura 4) es un pigmento vegetal liposoluble que se forma en las plantas superiores y se concentra mayoritariamente en las hojas, donde protegen a las células de la influencia dañina de la luz (Davidov-Pardo et al., 2016). Debido a su actividad colorante, este pigmento se caracteriza por ser utilizado en la industria de embutidos cárnicos de tipo Frankfurt. Autores como Granado-Lorencio et al. (2010) elaboraron productos cárnicos con adición de luteína $(4 \mathrm{mg} / 100 \mathrm{~g})$ enriquecida con rangos de concentraciones de grasa de $29-34 \% \mathrm{p} / \mathrm{p}$ y $73-81 \%$ $\mathrm{p} / \mathrm{p}$ en extracto de aceite de oliva $(4 \mathrm{~mL} / 100 \mathrm{~g})$, lo que permitió mejorar, durante el almacenamiento a $4^{\circ} \mathrm{C}$, los parámetros de color $\mathrm{a}^{*}$, luminosidad, textura y carotenoides totales. También, Hayes et al. (2010) evaluaron la adición de luteína (100-200 $\mu \mathrm{g} / \mathrm{g})$, sésamo (250-500 $\mu \mathrm{g} / \mathrm{g}$, ácido elágico (300-600 $\mu \mathrm{g} / \mathrm{g})$ y extracto de hoja de olivo (100-200 $\mu \mathrm{g} / \mathrm{g})$ en la formulación de empanadas de carne almacenadas durante 12 días a $4^{\circ} \mathrm{C}$ y encontraron que estos compuestos naturales mejoraron la estabilidad lipídica y las propiedades sensoriales en el producto cárnico.

Figura 4. Estructura de la luteína

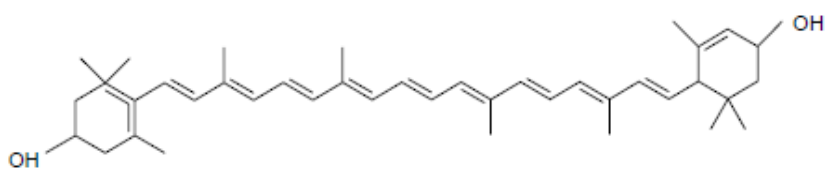

Fuente: elaboración propia.

\section{Betalaínas}

Las betalaínas son pigmentos que se extraen principalmente de la remolacha roja y se dividen en betacianinas (figura 5) y betaxantinas (Cordeiro et al., 2009). Debido a su color rojo y violeta característico, ha sido un pigmento de interés para la formulación de productos cárnicos como alternativa de colorante natural. En este marco, Bloukas et al. (1999) elaboraron salchichas con 18\% $\mathrm{p} / \mathrm{p}$ de grasa junto con adición de colorante natural E162 betanina y nitrito de sodio en cantidades de $150 \mathrm{mg} / \mathrm{Kg}$ y $100 \mathrm{mg} / \mathrm{Kg}$ respectivamente, lo que dio como resultado un incremento del enrojecimiento de la masa cárnica y una mejor aceptación hacia los consumidores. Adicionalmente, Zamudio-Flores et al. (2015) reportaron la adición de biopelículas de betalaínas en la elaboración de salchichas en condiciones de refrigeración a $4^{\circ} \mathrm{C}$ y $5 \%$ de humedad relativa, lo que dio como resultado una mejor conservación de los atributos de textura y color. 
Figura 5. Estructura de betacianina (betanina o rojo remolacha)

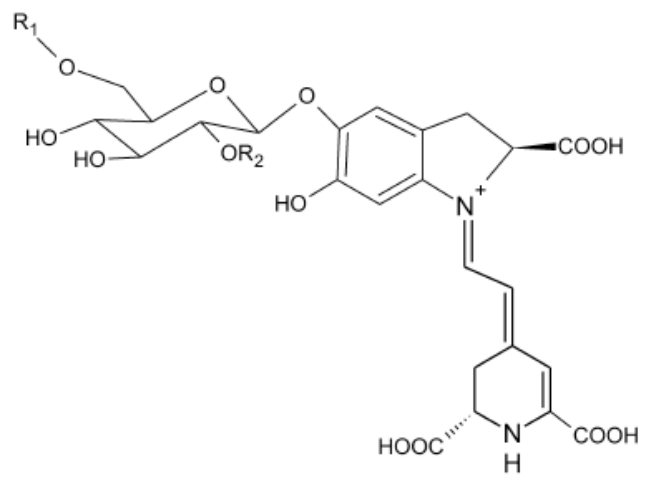

Fuente: elaboración propia.

\section{La Curcumina}

La curcumina (figura 6) es un colorante natural presente en el rizoma de la planta (Curcuma longa), añadido generalmente con otros compuestos. Al aprovechar la actividad colorante de la cúrcuma, Ceballos et al. (2012) realizaron varias microencapsulaciones por evaporación con solventes y coprecipitación, lo que les permitió concluir que la mejor relación para la obtención del colorante natural es de 1:1 en presencia de alfa-ciclodextrina mediante tratamiento de coprecitación en solución con agua y etanol.

Los anteriores autores lograron obtener un complejo de ciclodextrina-curcumina al obtener así un colorante sustituto del colorante artificial (tartrazina), como una alternativa de sustitución en productos cárnicos.

Figura 6. Estructura de la curcumina<smiles>COc1cc(/C=C/C(=O)CC(=O)/C=C/c2ccc(O)c(OC)c2)ccc1O</smiles>

Fuente: elaboración propia.

\section{COMPUESTOS NATURALES USADOS COMO ANTIOXIDANTES EN PRODUCTOS CÁRNICOS}

Los antioxidantes son sustancias que tienen la ca-

pacidad de retardar la oxidación biomolecular de compuestos fácilmente oxidables, tales como los lípidos y las proteínas en los productos cárnicos. Éstos tanto mejoran la vida útil de los mismos mediante la protección frente al deterioro causado por la oxidación como inhiben la producción de radicales libres (Bohm et al., 2002; Nassu et al., 2003). El uso de antioxidantes en los productos alimenticios está controlado por las leyes reguladoras del país fabricante o por normas internacionales. En la naturaleza se puede encontrar gran variedad de antioxidantes naturales en frutas y otros materiales vegetales que han sido utilizados como antioxidantes naturales, tales como el extracto de semilla de uva, hojas de cacao, hojas de olivo, extracto de corteza de romero, orégano, entre otras especias (Karre et al., 2013; Manzoor et al., 2014).La importancia de los antioxidantes en el metabolismo radica en que las células humanas constantemente están expuestas al ataque de radicales libres (RL), los cuales crean una serie de efectos negativos en el organismo como el envejecimiento. Por tal razón, el ser humano necesita de antioxidantes naturales como la vitamina $\mathrm{E}$, vitamina $\mathrm{C}$, carotenoides y compuestos fenólicos que se encuentran de forma natural en diversos tejidos vegetales como hojas, raíces, tallos, frutos, semillas y cortezas de plantas (Lee et al., 2006; González \& Vigoa, 2007; Trindade et al., 2010; Karre et al., 2013; Shah et al., 2014).

Dado los beneficios que presentan los antioxidantes naturales, se han convertido en compuestos de interés para ser evaluados en carnes y productos cárnicos. Una revisión de la literatura indica que las flores de litchi, las hojas de M.Oleifera y el extracto de zanahoria son una tendencia actual de aplicación como antioxidantes naturales en productos cárnicos.

\section{Flores de litchi}

Litchi (Litchi chinensis Sonn.) es una fruta tropical y subtropical de la familia Sapindaceae, y también un fruto importante en la economía de Taiwán (Ding et al., 2015). El fruto de litchi es comestible y se caracteriza por contener polifenoles entre los que se destacan el ácido gálico $(0.21 \mathrm{mg} / 100$ $\mathrm{mL})$, quercetina $(0.28 \mathrm{mg} / 100 \mathrm{~mL})$, ácido cafeico $(0.36 \mathrm{mg} / 100 \mathrm{~mL})$, 3-rutinósido (rutina), glucósido $(0.47 \mathrm{mg} / 100 \mathrm{~mL})$, catequina $(4.60 \mathrm{mg} / 100 \mathrm{~mL})$, epicatequina $(9.65 \mathrm{mg} / 100 \mathrm{~mL})$ y ácido gentísico $(10.95 \mathrm{mg} / 100 \mathrm{~mL})$ que pueden eliminar los radicales libres, y permitir la reducción de los valores de ácido tiobarbitúrico (TBARS) según lo reportado por Ding et al. (2015). 
A su vez, los anteriores autores evaluaron la adición de harina de flores de litchi en albóndigas de carne durante su almacenamiento en congelación $\left(-20^{\circ} \mathrm{C}\right)$ durante cuatro semanas en diferentes niveles de concentración $(0.5,1.0$ y $1.5 \%$ p/p). Los resultados evidenciaron que la adición de flores de litchi mejoró la textura y la capacidad de retención de agua de las albóndigas. A su turno, se presentó una tonalidad más roja y oscura frente al control. Los autores concluyeron que la adición de harina de flores de litchi en una concentración de $0.5 \% \mathrm{p} / \mathrm{p}$ presentó la mejor aceptación sensorial con un valor de 3.7 según la evaluación de los panelistas en relación con el sabor, olor y color; sin embargo, la adición de $1.5 \% \mathrm{p} / \mathrm{p}$ permitió la mayor reducción de ácido tiobarbitúrico con un valor de $86.97 \mathrm{eq} / \mathrm{g}$ que logró reducir la oxidación de lípidos y proteínas en el producto cárnico.

\section{Hojas de moringa}

Las hojas del árbol de la moringa (M. Oleifera) son ricas en antioxidantes, las cuales contienen un contenido fenólico de $24 \mathrm{mg}$ de ácido gálico/g de peso seco según lo reportado por Sreelatha y Padma (2009), quienes encontraron que el extracto de hojas de moringa $(750 \mathrm{mg} / \mathrm{kg})$ reduce significativamente los radicales DPPH (2,2-difenil-2-hidrato de picrilhidrazil). En relación con su aplicación en productos cárnicos, Jayawardana et al. (2015) evaluaron la adición de hojas de moringa en polvo en concentraciones de 0.75 y $1.00 \% \mathrm{p} / \mathrm{p}$ en salchichas de pollo. Los resultados evidenciaron que las incorporaciones del extracto de moringa mostraron valores significativamente inferiores de ácido tiobarbitúrico durante el almacenamiento en comparación con las salchichas de pollo elaboradas con el antioxidante sintético butilhidroxitolueno (BHT). Los autores manifiestan que la reducción de la concentración de ácido tiobarbitúrico $(1.6$ a $1.3 \mathrm{mg} /$ $\mathrm{kg}$ ) se debe a la inhibición de la peroxidación lipídica causada por las hojas de moringa que contienen polifenoles con efectos antioxidantes benéficos. La actividad antioxidante de los polifenoles se debe principalmente a sus propiedades redox, que pueden jugar un papel importante en la adsorción y neutralización de los radicales libres al bloquear el oxígeno singlete y a los peróxidos en degradación.

\section{Extracto de zanahoria}

La zanahoria (Daucus carota) es uno de los tubérculos importantes por su alto valor nutritivo dada su composición saludable de fitonutrientes y minerales. Es una buena fuente de antioxidantes naturales especialmente los carotenoides y compuestos fenólicos. Debido a estas propiedades, ciertos autores se han interesado en aplicar zumo de zanahoria en diferentes concentraciones a salchichas crudas durante el almacenamiento, refrigerado y congelado. En tal sentido, Badr y Mahmoud (2011) evaluaron salchichas con la adición de extracto de zanahoria en concentraciones de 19.843, 35 y $60 \%$ $\mathrm{p} / \mathrm{p}$ respectivamente mediante la aplicación de irradiación con radiación gamma en dosis de 0.3 y 4.5kGy. Los autores encontraron que la formulación de salchichas con el jugo de zanahoria que se concentró por 35 y $60 \%$ disminuyó la formación de hidroperóxidos entre $39.66-39.95 \% \mathrm{p} / \mathrm{p}$, a su vez disminuyó la formación de ácido tiobarbitúrico por $40.45 \%$ y $54.45 \% \mathrm{p} / \mathrm{p}$ después de los 60 días de almacenamiento en congelación para embutidos irradiados a 0.3 y $4.5 \mathrm{kGy}$.

Los resultados permitieron concluir que la aplicación de irradiación aumentó la concentración de ácido tiobarbitúrico en todos los casos; sin embargo disminuyó la presencia de la flora microbiana en la masa cárnica. Por otra parte, la concentración del jugo de zanahoria aumentó significativamente el contenido de compuestos antioxidantes naturales en el almacenamiento de las salchichas, debido a la presencia de los polifenoles presentes en la fruta. A su vez, Ekici et al. (2015) evaluaron salchichas fermentadas con adición de extracto de zanahoria y vieron un incremento en el contenido de compuestos fenólicos de $785.94 \mathrm{mg}$ de ácido gálico/Kg hasta $810.88 \mathrm{mg}$ de ácido gálico/Kg.

Finalmente, en la tabla 1 se presentan otros compuestos naturales de gran interés aplicados como colorantes y/o antioxidantes en productos cárnicos. 
Tabla 1. Otros colorantes y/o antioxidantes naturales

\begin{tabular}{|c|c|c|c|}
\hline PRODUCTO & COMPUESTO NATURAL & HALLAZGO & REFERENCIA \\
\hline $\begin{array}{l}\text { Carne de pollo } \\
\text { deshuesada }\end{array}$ & $\begin{array}{l}\text { Extracto de hojas de cacao }(200 \\
\text { a } 400 \mathrm{mg} / \mathrm{Kg})\end{array}$ & $\begin{array}{l}\text { Aumentó el poder antioxidante de la } \\
\text { carne al disminuir la peroxidación. }\end{array}$ & Hassan y Fan (2005) \\
\hline $\begin{array}{l}\text { Empanada de carne } \\
\text { de res }\end{array}$ & $\begin{array}{l}\text { Extracto de hojas de olivo ( } 100 \\
\text { a } 200 \mu \mathrm{g} / \mathrm{g})\end{array}$ & $\begin{array}{l}\text { Aumentó el poder antioxidante, } \\
\text { mejoró el color y la estabilidad lipídica } \\
\text { al disminuir los valores de ácido } \\
\text { tiobarbitúrico. }\end{array}$ & Hayes et al. (2010) \\
\hline $\begin{array}{c}\text { Hamburguesa } \\
\text { de carne de cabra }\end{array}$ & $\begin{array}{l}\text { Extracto de corteza de } \\
\text { mandarina }(10 \mathrm{~mL} / 2 \mathrm{Kg} \text { de } \\
\text { carne) }\end{array}$ & $\begin{array}{l}\text { Aumentó el contenido de compuestos } \\
\text { fenólicos totales en el producto final y } \\
\text { mejoró los parámetros de color en el } \\
\text { almacenamiento. }\end{array}$ & Devatkal et al.(2010) \\
\hline Salchicha de bologna & $\begin{array}{l}\text { Aceite esencial de romero } \\
(0.02 \% \mathrm{p} / \mathrm{p})\end{array}$ & $\begin{array}{l}\text { Aumentó la vida útil del producto } \\
\text { empacado al vacío al reducir la } \\
\text { peroxidación. }\end{array}$ & Viuda-Martos et al.(2010) \\
\hline $\begin{array}{l}\text { Hamburguesa de carne } \\
\text { de pollo }\end{array}$ & $\begin{array}{l}\text { Jugo del fruto de granada }(1: 2 \\
\text { carne/jugo } \mathrm{p} / \mathrm{p})\end{array}$ & $\begin{array}{l}\text { Mejoró el color del producto, aumentó } \\
\text { los poli- fenoles y no se presentaron } \\
\text { alteraciones microbianas en el } \\
\text { almacenamiento. }\end{array}$ & $\begin{array}{l}\text { Vaithiyanathan } \\
\text { et al.(2011) }\end{array}$ \\
\hline $\begin{array}{l}\text { Nuggets de carne de } \\
\text { cabra }\end{array}$ & $\begin{array}{l}\text { Extracto de polvo de brócoli }(1, \\
1.5 \text { y } 2 \% \mathrm{p} / \mathrm{p})\end{array}$ & $\begin{array}{l}\text { presentó un comportamiento similar al } \\
\text { BHT entre } 50 \text { y } 100 \text { ppm. }\end{array}$ & Banerjee et al. (2012) \\
\hline $\begin{array}{l}\text { Salchichones } \\
\text { cantoneses }\end{array}$ & $\begin{array}{l}\text { Extracto de semillas de loto } \\
(6.25,12.5,25,50 \mathrm{y} \\
100 \mu \mathrm{g} / \mathrm{mL})\end{array}$ & $\begin{array}{l}\text { Disminuyó el índice de peróxidos y el } \\
\text { valor de la acidez en el producto final. }\end{array}$ & Qi y Zhou (2013) \\
\hline Salchichas Frankfurt & $\begin{array}{l}\text { Extracto oleoso de residuos de } \\
\text { chontaduro ( } 9 \text { a } 97 \mathrm{~mL} / \mathrm{Kg} \text { ) }\end{array}$ & $\begin{array}{l}\text { Se disminuyó el contenido de adición de } \\
\text { nitratos y se mejoró la coordenada de } \\
\text { color } b^{*}\end{array}$ & $\begin{array}{l}\text { Pinzón-Zárate et al. } \\
\text { (2015) }\end{array}$ \\
\hline Salchichas de cerdo & $\begin{array}{l}\text { Extracto liofilizado de polen de } \\
\text { abeja }(10 \mathrm{~g} / 100 \mathrm{~mL})\end{array}$ & $\begin{array}{l}\text { Se disminuyó la oxidación lipídica en el } \\
\text { producto final y se incrementaron los } \\
\text { poli- fenoles }\end{array}$ & Almeida et al. (2016) \\
\hline
\end{tabular}

Fuente: elaboración propia.

\section{CONCLUSIONES}

Los pigmentos vegetales y gran parte de los compuestos naturales tienen diversas aplicaciones en la industria cárnica, como colorantes y antioxidantes, por lo cual es fundamental fortalecer nuevos mecanismos e investigaciones sobre otras fuentes de obtención de pigmentos y/o compuestos naturales. En Colombia, la investigación sobre compuestos naturales aplicados en productos cárnicos no es muy notoria. Lo anterior posibilita la búsqueda de nuevas investigaciones en este campo que contribuyan a buscar aditivos naturales que sean saludables y nutritivos. En esta revisión se presentaron algunos de los pigmentos y compuestos naturales que han logrado mantener la actividad oxidativa y el color en la elaboración de embutidos cárnicos. En este sentido, la utilización de los antioxidantes naturales en productos cárnicos permite minimizar los cambios oxidativos; es por esto, que todas las investigaciones reportadas en esta revisión se centraron en la búsqueda de nuevos antioxidantes y/o colorantes procedentes de fuentes naturales, en particular de origen vegetal.

\section{REFERENCIAS}

Almeida, J., Soares, R., Serafini, H., Pereira, D., Bianchin, M., Moura, C., Plata-Oviedo, V., Windson, I., Ribeiro, S., Pinto, F., y Teresinha, C. (2016). Lyophilized bee pollen extract: A natural antioxidant source to prevent lipid oxidation in refrigerated sausages. LWT-Food Science and Technology, In Press, 1-7.

Badr, H. \& Mahmoud, K. (2011). Antioxidant activity of carrot juice in gamma irradiated beef sausage during refrigerated and frozen storage. Food Chemistry, $1119-1130$. 
Banerjee, R., Verma, A., Das, A., Rajkumar, V., Shewalkar, A., \& Narkhede, H. (2012). Antioxidant effects of broccoli powder extract in goat meat nuggets. Meat Science, 179 -184 .

Barringer, S., Abu-Ali, J., \& Chung, H. (2005). Electrostatic powder coating of Sodium erythorbate and GDL to improve color and decrease microbial counts on Meat. Innovative. Food Science and Emerging Technologies, 6, 189-193.

Bener, M., Özyürek, M., Güc, lü, K., \& Apak, R. (2010). Polyphenolic contents of natural dyes produced from industrial plants assayed by HPLC and novel spectrophotometric methods. Industrial Crops and Products,32, 499-506.

Biswas, A., Chatli, M., \& Sahoo, J. (2012). Antioxidant potential of curry (Murraya koenigii L.) and mint (Mentha spicata) leaf extracts and their effect on colour and oxidative stability of raw ground pork meat during refrigeration storage. Food Chemistry, 467-472.

Boo, H., Hwang, S., Bae, C., Park, S., Heo, B., \& Gorinsteine, S. (2012). Extraction and characterization of some natural plant pigments. Industrial Crops and Products, 40, 129-135.

Bohm, V., Puspitasari-Nienaber, N., Ferruzzi, Ferruzzi, M., \& Schwartz, S. (2002) (2002). Trolox equivalent antioxidant capacity of different geometrical isomers of a-carotene, B-carotene, lycopene, and zeaxanthin. Journal of Agricultural and Food Chemistry, 50, 221-226.

Bojórquez, R., Gallego, J., y Collado, P. (2013).Propiedades funcionales y beneficios para la salud del licopeno. Nutrición Hospitalaria, 28, 6-15.

Bloukas, J., Arvanitoyannis, I., \& Siopi, A. (2015).. Effect of natural colorants and nitrites on colour attributes of frankfurters. Meat Science, 52, 257-265.

Burdulis, D., Sarkinas, A., Jasutiené, I., Stackevicené, E., Nikolajevas, L., \& Janulis, V. (2009). (2009). Comparative study of anthocyanin composition, antimicrobial and antioxidant activity in bilberry (Vaccinium myrtillus L.) and blueberry (Vaccinium corymbosum L.) fruits. Acta Poloniae Pharmaceutica, 66, 399-408.
Cabrita, L., Fossen, T., \& Andersen, O. (2000). Colour and stability of the six common anthocyanidin 3-glucosides in aqueous solutions. Food Chemistry,68(1), 101-107.

Calvo, M., García, M., \& Selgas, M. (2008). Dry fermented sausages enriched with lycopene from tomato peel. Meat Science, 80, 167172.

Ceballos, J., Lopez-Padilla., Caicedo, J., \& Cano, S. (2012). Obtaining cyclodextrin-curcumin complex and its use as a substitute of tartrazine. Revista Lasallista de Investigación, 9(2), 75-86.

Cordeiro, H., Pereira, A., Rodrigues, A., Gouveia, S., \& Méndez, K. (2009). Study on efficiency of betacyanin extraction from red beetroots. International Journal of Food Science and Technology, 44, 2464-2469.

Choski, P., \& Joshi, V. (2007). A review on lycopene-extraction, purification, stability and applications. International Journal of Food Properties, 10, 289-298.

Davidov-Pardo, G., Ekin, C., \& McClements, D. (2016). Lutein-enriched emulsion-based delivery systems: influence of $\mathrm{pH}$ and temperatura on physical and chemical stability. Food chemistry, 196, 821-827.

Deda, M., Bloukas, J., \& Fista, G. (2007). Effect of tomato paste and nitrite level on processing and quality characteristics of frankfurters. Meat Science, 76, 501-508.

Devatkal, S., Narsaiah, K., \& Borah, A. (2010). Anti-oxidant effect of extracts of kinnow rind, pomegranate rind and seed powders in cooked goat meat patties. Meat Science,155-159.

Ding, Y., Wang, S., Yang, D., Chang, M., \& Chen, Y. (2015). Alleviative effects of litchi (Litchichinensis Sonn.) flower on lipid peroxidation and protein degradation in emulsified pork meatballs. Journal of Food and Drug Analysis, 23(3), 501-508.

Doménech-Asensi, G., García-Alonso, F., Martínez, E., Santaella, M., Martín-Pozuelo, G., Bravo, S., \& Periago, M. (2013). Effect of the addition of tomato paste on the nutritional 
and sensory properties of mortadella. Meat Science, 93, 213-219.

Ekici, L., Ismet, O., Safa, K., Oznur, C., Fatih, T., Osman, S., \& Hasan, Y. (2015). Effects of black carrot concentrate on some physicochemical, textural, bioactive, aroma and sensory properties of sucuk, a traditional Turkish dry-fermented sausage. LWT- Food Science and Technology, 62, 718-726.

Falowo, A., Fayemin, P., \& Muchenje, V. (2014). Natural antioxidants against lipid-protein oxidative deterioration in meat and meat products: A review. Food Research International, 171181.

Fernández, L., Pérez-A., J., Sayas, M., \& Lopezsantove, $\tilde{N}$. (2002). Effect of paprika (Capsicum annum) on color of spanish-type sausages during the resting stage. Journal of Food Science,67(6), 2410-2414.

García, L., Calvo, M., \& Selgas, D. (2009). Beef hamburgers enriched in lycopene using dry tomato peel as an ingredient. Meat Science, 83, 45-49.

Garzón, M., Londoño, L., Hurtado, M., Cardona, L., y Rincón, C. (2012).Aplicación de la páprika extraída por fluidos supercríticos y microencapsulada por spray-drying en un producto embutido. Una alternativa como colorante natural. Revista Lasallista de Investigación, 9, 87-101.

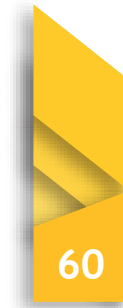

Garzón, A. (2008). Las antocianinas como colorantes naturales y compuestos bioactivos: Revisión. Acta Biológica Colombiana, 13(3), 27-36.

Granado-Lorencio, F., López, L., Herrero, B., Blanco, N., Cofrades, S., Pérez, S., Delgado, P., \& Jiménez, C. (2010). Lutein-enriched frankfurter-type products: Physicochemical characteristics and lutein in vitro bioaccessibility. Food Chemistry, 120, 741-748.

González, E., y Vigoa, Y. (2007). Colorantes naturales de origen vegetal.Ciencia y Tecnología de Alimentos, 17(1), 68-74.

Gómez, R., Ortiz, M., \& Pardo, J. (2008). Influence of the paprika type on redness loss in red line meat products. Meat Science, 80, 823-828.
Hassan, O., \& Fan, L. (2005). The anti-oxidation potential of polyphenol extract from cocoa leaves on mechanically deboned chicken meat (MDCM). LWT-Food Science and Technology, 38, 315-321.

Hayes, J., Stepanyan, V., Allen, P., O'Grady, M., \& Kerry, J. (2010). Effect of lutein, sesamol, ellagic acid and olive leaf extract on the quality and shelf-life stability of packaged raw minced beef patties. Meat Science, 84(4), 613-620.

Honikel, K. (2008). The use and control nitrate and nitrite for the processing of Meat products. Meat Science, 78, 68-76.

Hurtado, N., y Perez, M. (2014). Identificación, estabilidad y actividad antioxidante de las antoxianinas aisladas de la cascara del fruto de capulí. Información Tecnológica, 25(4), 131-140.

Jayawardana, B., Lalantha, N., \& Iddamalgoda, S. (2015). Weththasinghe Pabodha. Antioxidant and antimicrobial activity of drumstick (Moringa oleífera) leaves in herbal chicken sausages. LWT-Food Science and Technology, 1204-1208.

Jensen, M., Lopez, C., Payet, R., Liu, X., \& Konczak, I. (2011). Influence of copigment derived from Tasmannia pepper leaf on Davidson's plum anthocyanins. Journal of Food Science, 76, 447-453.

Jiménez, A., Chávez S., Verdalet, G., Iñigo, M., y Aquino, B. (2014). Betalaínas, polifenoles y actividad antioxidante en tuna roja mínimamente procesada, almacenada en atmósferas controladas. Gayana. Botánica, 71(2), 222-226.

Jiang, J., \& Xiong, Y. (2016). Natural antioxidants as food and feed additives to promote health benefits and quality of meat products: A review. Meat Science, 120,107-117.

Karre, L., Lopez, K., \& Getty, K. (2013). Natural antioxidants in meat and poultry products. Meat Science, 220-227.

Keeton, J., Rhee, K., Hafley, B., Nuñez, M., Boleman, R., \& Movileanu, I. (2002). Evaluation of plum/prune ingredients as a component 
of meat products part II. California Prune Board, 2-37.

Kong, J., Chia, L., Koh, N., Chia, T., \& Brouillard, R. (2003). Analysis and biological activities of anthocyanins. Phytochemistry, 64, 923-933.

Lazze, M., Savio, M., Pizzala, R., Cazzalini, O., Perucca, P., Scovassi, A., Stivala, L., \& Bianchi, L. (2004). Anthocyanins induce cell cycle perturbations and apoptosis in different human cell lines. Carcinogenesis, 25, 1427-1433.

Lee, S., Faustman, C., Djordjevic, D., Faraji, H., \& Decker, E. (2006).Effect of antioxidants on stabilization of meat products fortified with $\mathrm{n}-3$ fatty acids. Meat Science, 18-24.

Mercadante, A., Capitani, C., Decker, E., \& Castro, I. (2010). Effect of natural pigments on the oxidative stability of sausages stored under refrigeration. Meat Science, 84, 718-726.

Nassu, R., Goncalves, G., Pereira, A., \& Beserra, F.J. (2003). Oxidative stability of fermented goat meat sausage with different levels of natural antioxidant. Meat Science, 43-49.

Osterlie, M., \& Lerfall, J. (2005). Lycopene from tomato products added minced meat: Effect on storage quality and colour. Food Research International, 38, 925-929.

Ortíz, M., Vargas, M., Madinaveitia, R., \& Velázquez, J. (2011). Propiedades funcionales de las antocianinas. Revista de Ciencias Biológicas y de la Salud, XIII, 16-22.

Pinzón-Zárate, L., Hleap-Zapata, J., y Ordóñez-Santos, L. (2015). Análisis de los parámetros de color en salchichas Frankfurt adicionadas con extracto oleoso de residuos de chontaduro (Bactris gasipaes). Información Tecnológica, 26(5), 45-54.

Qi, S., \& Zhou, D. (2013). Lotus seed epicarp extract as potential antioxidant and anti-obesity additive in Chinese Cantonese Sausage. Meat Science, 257-262.

Revilla, I., \& Quintana, A. (2005). The effect of different paprika types on the ripening process and quality of dry sausages. International Journal of Food Science and Technology, 40, 411-417.
Rey, J., Martínez, C., \& Urrea, A. (2011). Evaluation of sensory characteristics and texture of an economic Buffalo meat (Bubalus bubalis) sausage and an economic beef (Bos indicus) sausage with addition of bovine hemoglobin poder. Procedia Food Science, 1, 545-548.

Rodríguez-Amaya, D. (2015). Carotenes as and xanthophylls antioxidants. Food Science, Technology and Nutrition, 17-50.

Sánchez, J. (2013). La química del color en los alimentos. Química Viva, 12(3), 234-246.

Selani, M., Contreras-Castillo, C., Shirahigue, L., Gallo, C., Plata-Oviedo, M., \& Montes-Villanueva, N. (2011). Wine industry residues extracts as natural antioxidants in raw and cooked chicken meat during frozen storage. Meat Science, 88, 397-403.

Sreelatha, S., \& Padma, P. (2009). Antioxidant Activity and Total Phenolic Content of Moringa oleifera Leaves in Two Stages of Matur. Plant Foods for Human Nutrition, 64(4), 303-311.

Singh, R., Jain, A., Panwar, S., Gupta, D., \& Khare, S. (2005). Antimicrobial activity of some natural dyes. Dyes and Pigments, 66, 99-102.

Trindade, R., Mancini, F., \& Villavicencio, A. (2010). Natural antioxidants protecting irradiated beef burgers from lipid oxidation. LWT-Food Science and Technology, 98-104.

Vaithiyanathan, S., Naveena, B., Muthukumar, M., Girish, P., \& Kondaiah, (2011). Effect of dipping in pomegranate (Punica granatum) fruit juice phenolic solution on the shelf life of chicken meat under refrigerated storage (4 $\left.{ }^{\circ} \mathrm{C}\right)$. Meat Science, 409-414.

Valenzuela, C., y Pérez, P. (2016). Actualización en el uso de antioxidantes naturales derivados de frutas y verduras para prolongar la vida útil de la carne y productos cárneos. Revista Chilena de Nutrición, 43(2), 188-195.

Viuda-Martos, M., Ruiz N., Fernandez, L., \& Perez-Alvarez, J. (2010). Effect of orange dietary fiber, oregano oil and packaging conditions on shelf-life of bologna sausages. Food Control, 21, 436-443. 
Zamudio-Flores, P., Ochoa, R., Ornelas-Paz., Saguilán, A., Vargas, T., Bello-Pérez, L., Rubio, R., \& Cárdenas-Félix, R. (2015). Effect of storage time on physicochemical and textural properties of sausages covered with oxidized banana starch film with and without betalains. CyTA - Journal of Food, 13(3), 456-463.
Zhoh, C., Kwon, H., \& Ahn, S. (2010). Antioxidative and antimicrobial effects to skin flora of extracts from peel of Allium cepa L. Asian Journal of Beauty \& Cosmetology, 8, 49-58. 